\title{
In situ nanoindentation during electrochemical hydrogen charging: a comparison between front-side and a novel back-side charging approach
}

\author{
M. J. Duarte ${ }^{1, *}$ (D), X. Fang ${ }^{1,2}$, J. Rao ${ }^{1}$, W. Krieger ${ }^{1}$, S. Brinckmann ${ }^{1,3}$, and G. Dehm ${ }^{1}$ (I) \\ ${ }^{1}$ Max-Planck Institut für Eisenforschung GmbH, 40237 Düsseldorf, Germany \\ ${ }^{2}$ Department of Materials and Earth Sciences, Technical University of Darmstadt, 64287 Darmstadt, Germany \\ ${ }^{3}$ Microstructure and Properties of Materials (IEK-2), Forschungszentrum Jülich, 52425 Jülich, Germany
}

Received: 25 August 2020

Accepted: 28 December 2020

Published online:

27 January 2021

(C) The Author(s) 2021

\begin{abstract}
The effects of hydrogen in metals are a pressing issue causing severe economic losses due to material deterioration by hydrogen embrittlement. A crucial understanding of the interactions of hydrogen with different microstructure features can be reached by nanoindentation due to the small volumes probed. Even more, in situ testing while charging the sample with hydrogen prevents the formation of concentration gradients due to hydrogen desorption. Two custom electrochemical cells for in situ testing were built in-house to charge the sample with hydrogen during nanoindentation: "front-side" charging with the sample and the indenter tip immersed into the electrolyte, and "back-side" charging where the analyzed region is never in contact with the solution. During front-side charging, surface degradation often occurs which also negatively influences analyses after hydrogen charging. The back-side charging approach proposed in this work is a promising technique for studying in situ the effects of hydrogen in alloys under mechanical loads, while completely excluding the influence of the electrolyte on the nanoindented surface. Hydrogen diffusion from the charged back-side toward the testing surface is here demonstrated by Kelvin probe measurements in ferritic FeCr alloys, used as a case study due to the high mobility of hydrogen in the bcc lattice. During nanoindentation, a reduction on the shear stress necessary for dislocations nucleation due to hydrogen was observed using both setups; however, the quantitative data differs and a contradictory behavior was found in hardness measurements. Finally, some guidelines for the use of both approaches and a summary of their advantages and disadvantages are presented.
\end{abstract}

Handling Editor: M. Grant Norton.

Address correspondence to E-mail: j.duarte@mpie.de 


\section{GRAPHICAL ABSTRACT}

\section{In-situ nanoindentation of hydrogen charged alloys}

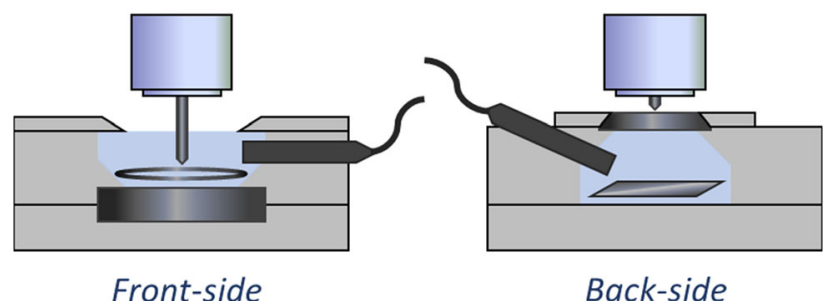

\section{Introduction}

Hydrogen represents a genuine opportunity for the generation of low-carbon-emission energy with the development of fuel cells [1, 2]. However, hydrogeninduced mechanical degradation of materials, or hydrogen embrittlement, is also a problem causing significant worldwide economic losses [3-6]. The research on hydrogen therefore aims either to profit from the hydrogen evolution reaction (HER) or to prevent hydrogen-related failure. One of the most demanding challenges is the direct characterization of hydrogen and its impact on materials due to its small size often leading to high diffusivity. Material failure initiates at the atomic scale by hydrogen interactions with trapping sites, such as dislocations, grain/phase boundaries or precipitates, as depicted in Fig. 1. In these processes, numerous factors are involved (material characteristics, mechanical state, environmental conditions), and defining a single

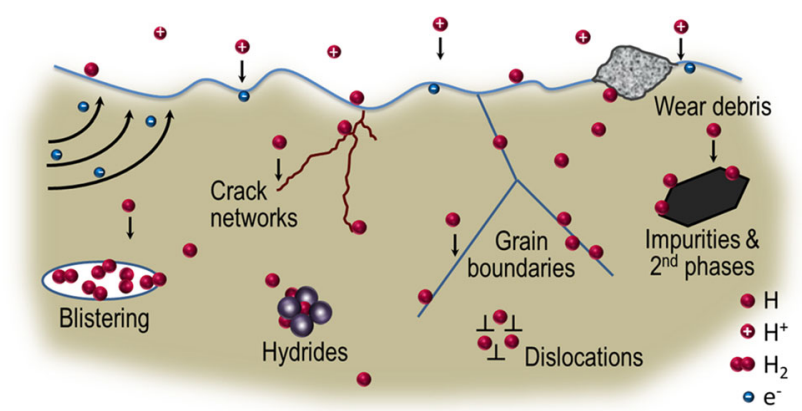

Figure 1 Schematic figure of the hydrogen diffusion process into a bulk alloy, depicting also the interaction of hydrogen with different features in material. failure mechanism is not possible. This is why often collaborative, complementary, competing or contradictory mechanisms have been proposed [6-10].

A study of the hydrogen-metal interactions at the nanometer and microstructure length scales is compulsory. Transmission electron microscopy (TEM) can provide direct evidence of the interplay of dislocations with hydrogen [11, 12], and more recently nanoindentation tests are being used to evaluate nucleation stresses for dislocations and the material hardness [13-16]. Nanoindentation allows independent characterization of small volumes in material to focus on single phases, interfaces or precipitates. However, in most cases, the samples are pre-charged ex-situ leading to hydrogen desorption prior and during testing. In situ nanoindentation during hydrogen charging was proposed in 2006 by Vehoff and Barnoush [15] under the term of "electrochemical nanoindentation", to study the effects of hydrogen on dislocation nucleation in (111) $\mathrm{Ni}$, and later in other materials [17-19]. These studies show a decrement in the maximum shear stress and dislocation mobility at cathodic charging potentials, suggesting also that hydrogen decreases the Gibbs free energy required for homogeneous dislocation nucleation. This effect and a reduction in the lattice cohesion with increased hydrogen content are also explained by the defactant concept in [13, 20].

In this work, we fabricated a custom electrochemical setup similar in concept to that from [15] and performed several tests to evaluate the viability of the technique to study the hydrogen effects particularly in bcc Fe alloys. This setup will be referred to as "front-side" charging from now on. As detailed 
hereafter, we designed and built a new setup based on a novel "back-side" charging approach, due to several drawbacks encountered during front-side charging, mainly related to surface changes occurring during electrochemical hydrogen charging of the Fe alloys. This novel back-side charging concept and its highlights were first presented in [21]. In this manuscript, we evaluate both setups while testing ferritic $\mathrm{Fe}-\mathrm{Cr}$ alloys. We provide guidelines on how to perform reliable in situ measurements using either setup and highlight their advantages and disadvantages.

$\mathrm{Fe}-\mathrm{Cr}$ alloys were chosen as a case study as they are the base elements in stainless steels, which are commonly used for the storage and transport of water, fluids, chemicals or vapors. The effect of hydrogen is especially severe in Fe due to its high mobility through interstitial sites. A high $\mathrm{Cr}$ content was chosen here to reduce corrosion during charging experiments. Additionally, $\mathrm{Fe}-\mathrm{Cr}$ forms a single phase of a ferritic solid solution (body-centered cubic, $b c c)$ and the formation of the undesired sigma phase is suppressed below 20 at.\% of Cr. Bcc Fe can incorporate only a small fraction of $\mathrm{H}$ at room temperature and pressure, as low as 2 parts per million (0.0002 at.\%), while hydrogen mobility is high due to a very low diffusion barrier; the diffusivity of hydrogen in pure bcc Fe is about $1.0 \times 10^{-4} \mathrm{~cm}^{2} \mathrm{~s}^{-1}$ [22]. Under these conditions, it is expected that hydrogen will also desorb rapidly from bcc Fe-Cr alloys and consequently ex-situ testing is not feasible as the nearsurface region quickly becomes hydrogen depleted, making these alloys an ideal candidate for in situ testing.

\section{Materials and methods}

Fe-15 wt $\% \mathrm{Cr}$ and $\mathrm{Fe}-20 \mathrm{wt} \% \mathrm{Cr}$ alloys (further referred to as $\mathrm{Fe}-15 \mathrm{Cr}$ and $\mathrm{Fe}-20 \mathrm{Cr}$ ) were prepared by vacuum induction melting and cast into $\mathrm{Cu}$ molds, using raw materials with purity higher than $99.9 \%$. The cast alloys were recrystallized by hot rolling at $1100{ }^{\circ} \mathrm{C}$, heat-treated at $1300{ }^{\circ} \mathrm{C}$ for $4 \mathrm{~h}$ in an $\mathrm{Ar}$ atmosphere, quenched in water and annealed for $1 \mathrm{~h}$ at $800{ }^{\circ} \mathrm{C}$. This treatment ensured a homogeneous microstructure with equiaxed grains of at least a few hundred micrometers in diameter. The alloys were cut to the desired geometry by electrical discharge machining: a block of $18 \times 18 \times 4 \mathrm{~mm}^{3}$ for the frontside charging and a 2-mm-thick disk of $13 \mathrm{~mm}$ in diameter for the back-side charging approach. The samples were ground up to 4000 grit $\mathrm{SiC}$ paper, polished up to $1 \mu \mathrm{m}$ diamond suspension and finally polished with colloidal silica for about $1 \mathrm{~h}$ to remove the remaining deformation layer.

Scanning electron microscopy (SEM) images of the alloy surfaces and imprints before and after testing were taken in a Zeiss Auriga ${ }^{\circledR}$ dual-beam workstation. Electron backscatter diffraction (EBSD) was performed in a JEOL JSM 6490.

The surface morphology was characterized by atomic force microscopy (AFM, Veeco Digital Instruments, DI3100S-1). Regions of $8.5 \mu \mathrm{m} \times 8.5 \mu \mathrm{m}$ in size were scanned in tapping mode at a rate of $0.5 \mathrm{~Hz}$ and analyzed in the Gwyddion software.

Nanoindentation tests at a strain rate of $0.05 \mathrm{~s}^{-1}$ were carried out using continuous stiffness measurements (CSM) with Berkovich indenters for a maximum load of $10 \mathrm{mN}$. In a load controlled instrument, as it is the G200 nanoindenter, a constant strain rate is defined as $\dot{\varepsilon}=\dot{h} / h \approx \dot{P} / 2 P$, following Lukas and Oliver [23].The calculated tip radius was $\sim 150 \mathrm{~nm}$ for the tip used in the front-side charging and $170 \mathrm{~nm}$ for the back-side charging. The hardness and reduced modulus reported here were taken at an indentation depth of $90-100 \mathrm{~nm}$ in all cases. Two setups for charging hydrogen electrochemically (front-side and back-side charging), consisting of threeelectrode electrochemical cells, were designed and adapted into the commercial Nanoindenter G200 from Agilent/Keysight/KLA technologies. The cell body was machined out of polychlorotrifluoroethylene (PCTFE). Commercial DRIREF-2SH micro-reference electrodes were purchased from World Precision Instruments (WPI). The counter electrodes consist of either a Pt ring placed about $1 \mathrm{~mm}$ above the sample for the front-side charging or a $1 \mathrm{~cm}^{2} \mathrm{Pt}$ plate placed $4 \mathrm{~mm}$ below the sample for the back-side charging approach, both supplied by Goodfellow with $99.9 \%$ purity. Special nanoindenter tips for immersion into the electrolyte were requested from Synton-MDP for the front-side charging. The tip shaft was extended up to $11 \mathrm{~mm}$, and epoxy resin covers the welds between the diamond tip and the shaft to prevent corrosion. To immerse the nanoindenter tip into the cell, an additional stage with vertical motion was installed on top of the nanoindenter $\mathrm{XY}$ stage. This stage consists of an $\mathrm{Al}$ baseplate $(10 \times 10 \mathrm{~cm})$ with a Thorlabs Mini Lab Jack L200/M 
driven by a stepper motor Nanotec ST2818L1006 and controller Nanotec SMCI33 USB, following [24]. The stage motion was referenced by an end switch. Additionally, for the back-side charging, a continuous Ar flow was introduced to provide an oxygen-depleted atmosphere and minimize hydrogen desorption during the nanoindentation tests. Further specific characteristics and measurements performed in each setup are detailed in the results section.

Kelvin probe (KP) measurements were used to evaluate the hydrogen diffusivity through the tested alloys in the back-side charging approach. After polishing, the sample was rinsed with $\mathrm{CCl}_{4}$ to remove organic contaminants adsorbed on the surface. A $100 \mathrm{~nm}$ Pd thin film was deposited on one sample side by physical vapor deposition (PVD, Leybold Univex 450) to act as a sensor for the hydrogen permeation analysis. The sample was placed on the custom back-side charging cell (hydrogen entry side), with the Pd coated side on the top (hydrogen exit side), and introduced into the KP chamber. The $100 \mu \mathrm{m}$ diameter tip of the KP was positioned over the sample to measure hydrogen-induced potential changes at the exit side. The KP chamber was purged with dry $\mathrm{N}_{2}$ gas during the measurements. More details on the KP approach can be found in $[25,26]$.

We use liquid sources as hydrogen precursors to promote electrochemically the HER [27] at room temperature and pressure. All solutions were prepared with deionized water (ion concentration $<10$ $\mu \mathrm{S})$. Front-side charging tests were conducted using a similar electrolyte to [19] consisting of a $0.05 \mathrm{M} \mathrm{Na}_{2}$ $\mathrm{SO}_{4}$ solution with $\mathrm{H}_{2} \mathrm{SO}_{4}$ to control a $\mathrm{pH}$ of $4-6$, further referred to as sulfate buffer solution. A $0.1 \mathrm{M}$ $\mathrm{NaOH}$ solution was used for back-side charging tests adding $20 \mathrm{mg} / \mathrm{L}$ of $\mathrm{As}_{2} \mathrm{O}_{3}$ to promote hydrogen absorption by the alloys. The prepared $\mathrm{NaOH}$ solution was mixed at $50{ }^{\circ} \mathrm{C}$ with $10 \mathrm{wt}$. $\%$ of Agar (bacteriological grade from VWR chemicals) to form a hydrogel. A Reference $600^{\mathrm{TM}}$ potentiostat from Gamry instruments enabled the HER. All potentials are referred with respect to the reference electrode as $\mathrm{V}_{\text {Ref. }}$

\section{Results and discussion}

$\mathrm{Fe}-\mathrm{Cr}$ alloys were used for a fair comparison between front-side and back-side hydrogen charging. Figure 2a shows a single ferritic structure indexed from


Figure 2 a Inverse pole figure map of the $\mathrm{Fe}-15 \mathrm{Cr}$ alloy. b Typical nanoindentation load-displacement curve without hydrogen charging (Hertz fitting highlighted in red). c Example of a linear seep voltammetry in the negative direction for the $\mathrm{Fe}$ $20 \mathrm{Cr}$ alloy using the back-side charging setup to identify the HER region. d Current measurements during potentiostatic charging at $1.45 \mathrm{~V}_{\text {Ref }}$ (red line) and $1.5 \mathrm{~V}_{\text {Ref }}$ (black line) for the back-side hydrogen charging of the $\mathrm{FeCr} 20$ alloy.

the EBSD patterns, and specific grain orientations were selected from the inverse pole figures. The shown grain size above $400 \mu \mathrm{m}$ allowed performing nanoindentation tests before and during hydrogen charging within the same grain. Additional misorientation maps were analyzed to ensure no remnant mechanical deformation occurred during sample grinding and polishing. The indented region can be considered dislocation-free in the analyzed volume, as the dislocation density of the annealed $\mathrm{Fe}-\mathrm{Cr}$, revealed by chemical etching, is lower than $10^{12} \mathrm{~m}^{-2}$ (in other words, the average dislocation spacing is less than $1 \mu \mathrm{m}$ ).

Nanoindentation tests were conducted before and during hydrogen charging using the designed electrochemical setups for front-side and back-side charging. Figure $2 b$ shows the obtained typical loaddisplacement curves. The first elastic loading precedes a discontinuous displacement or "pop-in". This first stage of plastic strain, characterized by nanoindentation, is associated with the homogeneous nucleation of dislocations [28-30] and supported by modeling of the plastic flow in bcc Fe [31]. Note that dislocation nucleation can occur under the dislocation-free volume in the material below the indenter tip at the location of highest shear stress or as well at surface asperities, where local stress concentrations 
assist in dislocation nucleation. For the first case, the maximum shear stress beneath the indenter at the yield point $\left(\tau_{\max }\right)$ was calculated according to Hertz as [32]:

$\tau_{\max }=0.31\left(\frac{6 E_{r}^{2}}{\pi^{3} R^{2}} P\right)^{1 / 3}$

where $P$ is the measured pop-in load, $E_{r}$ the calculated reduced modulus, and the tip radius $R$, is obtained from the Hertzian contact theory [33] (red fitted line in Fig. 2b).

The HER was promoted at a constant potential following linear sweep voltammetry tests (Fig. 2c) to reach a specific current density for hydrogen charging (Fig. 2d). The HER is observed as a continuous current rise during cathodic charging (negative applied potential in Fig. 2c).

\section{Front-side charging}

A schematic of the front-side charging setup is shown in Fig. 3. The region of the $\mathrm{Fe}-\mathrm{Cr}$ sample acting as a working electrode (hydrogen-charged area) is defined by an O-ring of $9 \mathrm{~mm}$ in diameter. A Pt ring above the sample acts as a counter electrode, while the micro-reference electrode is inserted from the cell side. The cell has a capacity for $\sim 2 \mathrm{ml}$ of electrolyte, which is in direct contact with the testing surface and the immersed custom long indenter tip. This setup follows a similar concept as the one developed by Barnoush et al. [15], while considering as well the cell design for in situ AFM at electrified interfaces [34]. PCTFE was chosen for the cell body as the optimum candidate to withstand the applied mechanical loads while being inert to the electrolytes used for charging and having no water absorption, unlike other polymers such as polytetrafluoroethylene (PTFE of $0.01 \mathrm{wt} \%$ after $24 \mathrm{~h}$ standard immersion test) or polyvinyl chloride (PVC gaining up to $0.4 \mathrm{wt} \%$ ).

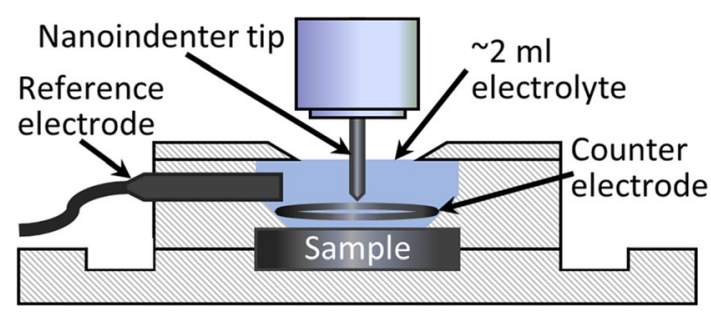

Figure 3 Schematic of the setup installed for front-side charging.
Water absorption can decrease Young's modulus of the polymer [35] and might affect the final data.

During nanoindentation of the $\mathrm{Fe}-15 \mathrm{Cr}$ alloy (EBSD from Fig. 2a), a reduction in the pop-in load was observed during cathodic charging, indicating a decrease in the maximum shear stress required for dislocation nucleation, as shown in the representative plot of Fig. 2a for the (4 34 ) orientation. Subsequent tests were made in the (lllll $\left.0 \begin{array}{ll}0 & 1\end{array}\right)$ and $\left(\begin{array}{lll}1 & 0 & 1\end{array}\right)$ grain orientations (Fig. 2b,c). As the typical signatures of a pop-in were not observed anymore, an anodic potential was applied to remove the influence of hydrogen in the testing, obtaining the curves for the (2 6 9) grain orientation of Fig. $2 \mathrm{~d}$. The cumulative probability of the pop-in load was extracted from the load-displacement curves of the $\left(\begin{array}{ll}4 & 3\end{array}\right)$ ) orientation and shown in Fig. 4e. This data suggests a decrement of the pop-in load of $82( \pm 23) \mu \mathrm{N}$ for the (4 3 4) grain orientation. Note that all the reference measurements were obtained in an air atmosphere followed by the addition of the electrolyte and consecutive nanoindentation tests for the different grain orientations under potentiostatic charging conditions. The alloy was initially pre-charged with hydrogen for $30 \mathrm{~min}$ before starting the nanoindentation tests and hydrogen charging continued as well during nanoindentation. This procedure was in accordance with reported tests in the field, such as in [17]. Similar hydrogen effects in the binary alloy Fe-15 wt.\% Cr were also addressed by in situ electrochemical nanoindentation in [19]. The experimental data was there correlated with linear elastic finite element calculations to estimate the critical shear stress necessary to nucleate dislocations in defect-free crystals. The authors reported a decrease in the critical shear stress between 10 and 20\% depending on the grain orientation, and associated the hydrogen enhanced dislocation nucleation with an increase in the mobile dislocation density and therefore with an initial softening of the material.

However, during our study, it is evident that chemical surface evolution during charging influenced the mechanical results, as they severely change in the charged condition as the test progresses according to the sequence of the indented grains: (4 3 4), (l $\left.\begin{array}{lll}0 & 0 & 1\end{array}\right)$ and finally ( $\left.\begin{array}{lll}1 & 0 & 1\end{array}\right)$. An initial reduction in the pop-in load of $48 \%$ for the (4 34 ) grain orientation could be associated with hydrogen effectively enhancing dislocation nucleation. However, a strong shift to unrealistically low load values is detected 

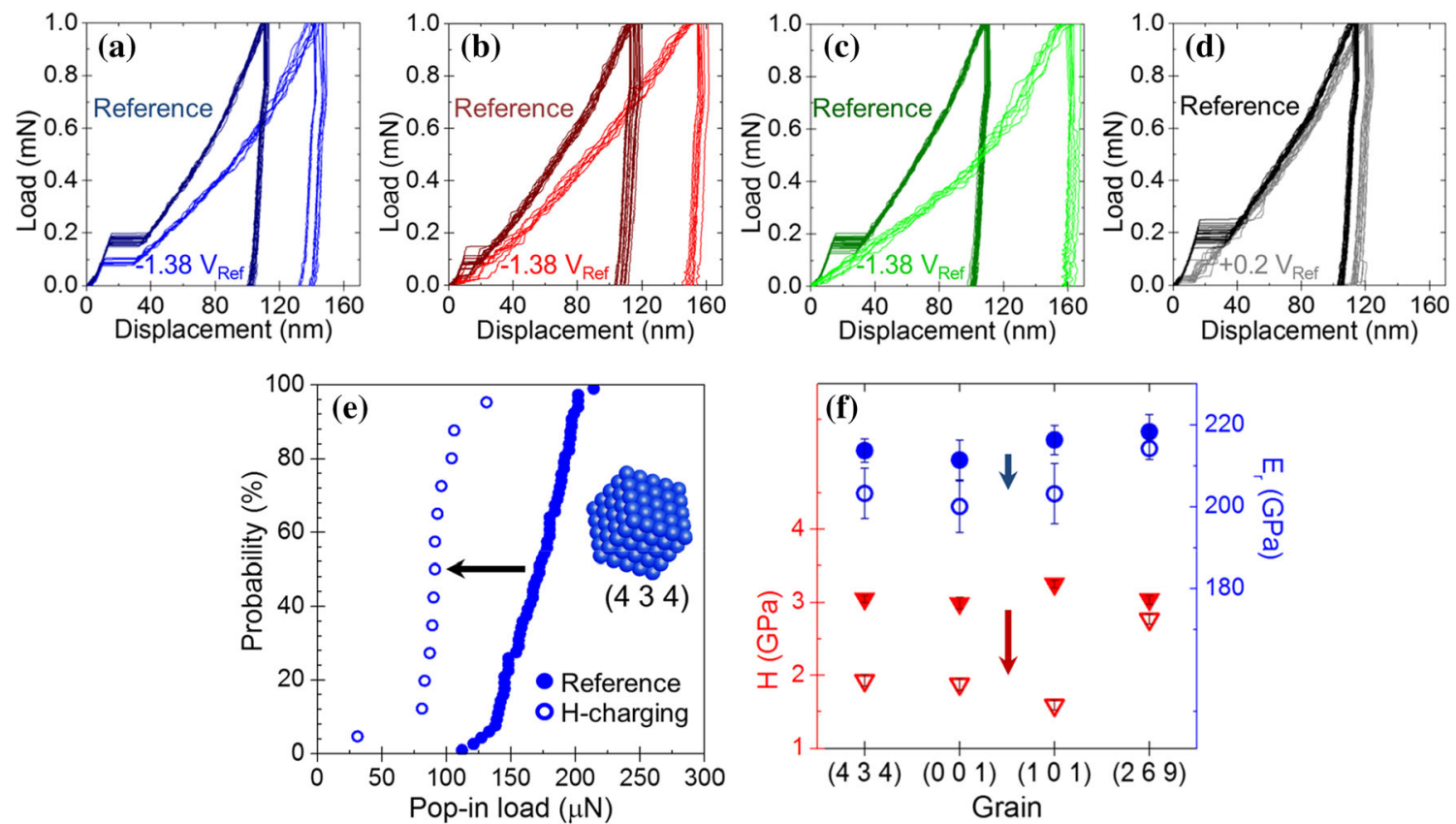

Figure 4 Front-side charging. a-d Nanoindentation curves for the $\mathrm{Fe}-15 \mathrm{Cr}$ alloy, obtained at different grain orientations before (Reference, in darker colors) and during potentiostatic charging at different potentials (brighter colors). e Probability distribution of the pop-in load for the (4 34 4) grain orientation according to

already for the $\left(\begin{array}{lll}0 & 0 & 1\end{array}\right)$ grain orientation. For the $\left(\begin{array}{lll}1 & 0 & 1\end{array}\right)$ grain orientation only seldom data points are observed at these low loads while most of the curves do not show a pop-in. These curves reveal plasticity from the beginning as load-displacement values are below the elastic Hertz prediction throughout the experiment. In addition, hardness and elastic modulus were calculated from the load-displacement curves according to Oliver and Pharr [36] and are shown in Fig. 4f. While applying an anodic potential (as in Fig. 4d for the (4 69 ) grain orientation), the initial hardness and modulus are partially recovered (Fig. 4f) and some unreliable pop-ins at less than $10 \mathrm{~nm}$ indentation depth are also recorded. However, a full recovery is not observed in this case. A reduction in the hardness (of 30-50\%) and the elastic moduli (5-10\%) are observed irrespective of the grain orientation. This behavior contradicts the findings using the back-side charging approach, which shows an increase in hardness and unchanged elastic modulus (see "Back-side charging" section). This contradictory data is for this particular case attributed to the interactions of the electrolyte in direct contact with the tested surface during the hydrogen charging process. The continuous contact of the electrolyte

Fig. 4a, before and during hydrogen charging in the sulfate buffer solution at $2 \mathrm{~mA} / \mathrm{cm}^{2}$ with $20 \mathrm{~min}$ pre-charging (the arrows indicate the shift of the curve with respect to the uncharged tests in air). f Measured hardness and reduced modulus before and during potentiostatic charging.

with the sample surface might promote the occurrence of one or more of the effects further detailed here depending on the particular electrolyte condition, charging parameters and sample immersion time.

The surface evolution due to charging was evaluated by performing reference indents as "markers", followed by AFM mapping such as in Fig. 5a. After cathodic polarization at $1 \mathrm{~mA}$ in the sulfate buffer solution for $60 \mathrm{~min}$, the initial surface roughness (of $<1 \mathrm{~nm}$ in average) increases up to $8 \mathrm{~nm}$, measured in the same location after polarization (Fig. $5 b$ ). This noticeable height profile change (Fig. 5c) is caused by cathodic corrosion during hydrogen charging. The specific charging conditions and the electrolyte purity might also lead to contamination or formation of particles or oxides on the materials surface, Fig. 5d. Surface mapping of other indent made in the reference state and subsequently exposed to hydrogen (Fig. 5e) reveals bulge-like features after polarization, up to $200 \mathrm{~nm}$ in diameter and $50 \mathrm{~nm}$ in height (Fig. 5f). These features, distributed homogeneously in the sample surface, resemble the formation of blisters due to the strong applied voltage. 

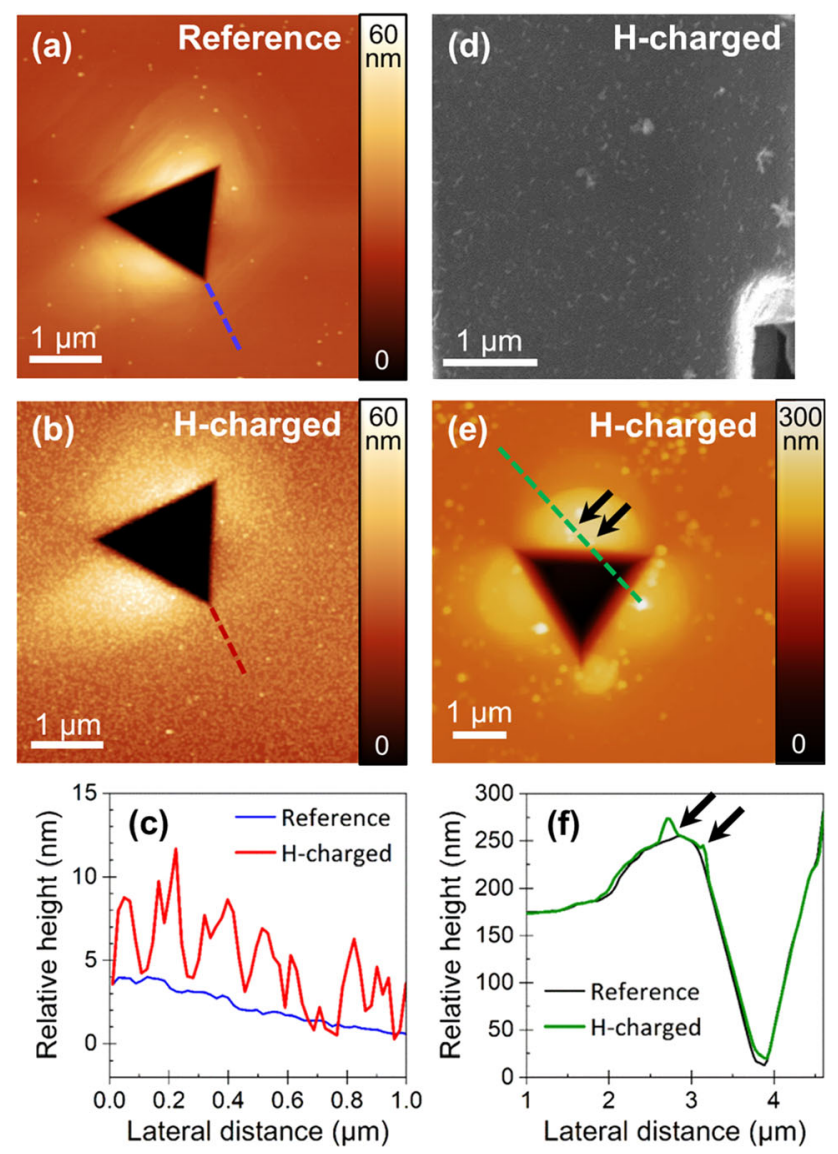

Figure 5 Front-side charging. a AFM map of a reference indent (no hydrogen charging) and $\mathbf{b}$ after applying a cathodic current of $1 \mathrm{~mA}$ for $30 \mathrm{~min}$; c relative height profile according to the blue and red dashed lines in $\mathbf{a}$ and $\mathbf{b}$. $\mathbf{d}$ SEM image of a sample surface after hydrogen charging at $-1.05 \mathrm{~V}_{\text {Ref }}$ for 60 min. e AFM map of an indent made in the uncharged state and mapped again after cathodic polarization at $-1.4 \mathrm{~V}_{\text {Ref }}$ for $30 \mathrm{~min}$, f Height profile along the green dashed line from e compared to the same location before hydrogen charging (black line).

This nanoscale surface evolution can be easily overseen when performing coarse characterization post-nanoindentation. An example is AFM-like scanning using nanoindentation tips [37] which are typically one order of magnitude larger in tip radius than AFM tips, therefore decreasing the resolution of the scan and neglecting possible surface reshaping at a small scale. As the pop-in load is highly sensitive to the surface conditions (since pop-in usually occurs at a depth of tens of nanometers), the increased surface roughness and step formation can explain the continuous pop-in load decrease during the testing of three consecutive grains shown here as local stress concentrations assist in dislocation nucleation.
Moreover, blister formation, surface contamination and increased oxides and hydroxides native layer might be the origin of the observed decrease in the hardness and elastic modulus.

Additional mechanical effects during front-side charging are introduced by the contact of the electrolyte with the long indenter tip, resulting in added capillary forces, viscous damping and vibrations introduced by the electrolyte flow. Hydrogen recombination to form $\mathrm{H}_{2}$, common for high charging potentials, leads to bubble formation, contact loss between the metal surface and the electrolyte, as well as mechanical vibrations. A liquid film is always present between the indenter tip and the sample surface, resulting in the formation of corrosion products that complicate the detection of the initial contact and deteriorate the data reliability at low indentation depths.

There have been several efforts directed to minimize the aforementioned challenges, as often one or more of them might occur while testing different materials. Here, we compile a short guide of recommendations for the use of the front-side charging approach and reliable data analysis based on our own experience and compiled literature:

- When possible, perform the frame stiffness calibration by directly mounting the reference material on the setup for hydrogen charging.

- Use the most suitable electrolyte to reduce surface damage, for example, a glycerol-based solution [37] or borate buffer electrolyte [38], while considering also the kinetics of the HER to determine the appropriate charging conditions.

- Perform the reference measurements (i.e., without hydrogen), with the sample and tip immersed already into the electrolyte. This accounts for capillary forces, surface tension and damping effects, allowing a reliable comparison of the mechanical behavior once hydrogen is introduced.

- Use a freshly polished sample for every set of measurements. For example, measure only onegrain orientation per test. The longer the sample remains in the electrolyte, the higher the surface damage.

- Optimize the charging current density, for example, $<2 \mathrm{~mA} / \mathrm{cm}^{2}$ for bcc FeCr alloys. The use of large current densities oversaturates the level of hydrogen at the sample surface and only increases 
the rate of $\mathrm{H}_{2(g)}$ formation, generating bubbles and vibrations on the indenter tip. Large current densities also increase surface damage [39]. The hydrogen amount that a material can absorb is limited by the material chemistry and microstructure itself.

- Perform dedicated AFM high-resolution maps of the surface prior and postmortem to detect features formed during the hydrogen charging process.

\section{Back-side charging}

In the back-side charging setup (Fig. 6) the cell is placed below the sample and hydrogen reaches the testing (upper) surface through diffusion in the bulk. This design can be understood as an inverted threeelectrode cell with respect to the front-side charging. The cell body is machined from PCTFE and the sample at the top (working electrode) is in this case a $2 \mathrm{~mm}$ thick disk of the Fe-20Cr alloy, with an area of $8 \mathrm{~mm}$ in diameter in contact with the electrolyte. About $7 \mathrm{ml}$ of electrolyte are introduced, in this case, a $0.1 \mathrm{M} \mathrm{NaOH}$ solution in hydrogel form. The hydrogel prevents losing contact between the electrolyte and the sample due to bubble formation during charging at the back/bottom side of the sample. This frequently occurs while using a liquid precursor without continuous electrolyte flow, which in turn might cause vibrations. The structural integrity of the polymer network is kept during the charging process and ensures continuous and remarkably stable hydrogen flow through the sample during several hours, as shown in Fig. 2 d.

For the back-side charging, nanoindentation tests were performed similarly to the ones in the front-side charging setup. The reference data (without hydrogen) was obtained with the cell fully loaded with the electrolyte but without applying any potential. Once the reference data is obtained, nanoindentation tests

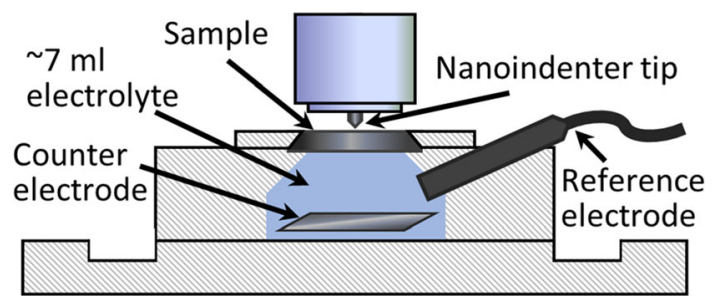

Figure 6 Schematic of the setup designed for back-side charging. are performed continuously at the same time that hydrogen is introduced at a constant potential equivalent to a current density of $\sim 2 \mathrm{~mA} / \mathrm{cm}^{2}$, during the complete test. The mechanical data in Fig. 7 were collected after $2 \mathrm{~h}$ during hydrogen charging conditions to reach a steady state described by the equilibrium between the hydrogen flow at the entry side and hydrogen desorption at the exit side. This was monitored by changes in the measured hardness until a plateau of stable values was reached. Nanoindentation tests in Fe-20Cr (Fig. 7a), for the (1 $10)$ grain orientation, show a mean reduction in the pop-in load for dislocations nucleation of $38 \mu \mathrm{N}$, indicating a decreased maximum shear stress of $\sim$ $11.1 \%$ with increasing hydrogen content (Fig. $7 b$ ). This is consistent with a multiscale simulation of homogeneous dislocation nucleation combining atomistic information (e.g., dislocation core structure, hydrogen-hydrogen interaction) and a self-consistent iterative method for the local hydrogen content in a dislocation stress field [40]. According to that approach, the reduced critical shear stress can be explained as an effective decrease of the dislocation line energy due to the interaction with diffusible hydrogen. An increase in hardness up to $17 \%$ (Fig. 7c) can be attributed to higher dislocation-dislocation interactions, as discussed in [41], related to the increased nucleation rate. In addition, the formation of hydrogen Cottrell atmospheres around the dislocation core could contribute to dislocations pinning [42, 43]. A full study dedicated to the hydrogen effect in $\mathrm{FeCr}$ alloys will be presented in a further publication.

Hydrogen permeation was evaluated by KP measurements to validate the presence of hydrogen in the analysis surface. The back-side charging setup was directly introduced into the KP chamber, and hydrogen charging was promoted by keeping a constant current density of $2 \mathrm{~mA} / \mathrm{cm}^{2}$. The recorded potential changes (Fig. $7 \mathrm{~d}$ ) were then used to calculate the time lag and the effective diffusion rate according to [26]. The calculated diffusion rate was estimated as $1.5 \times 10^{-6} \mathrm{~cm}^{2} / \mathrm{s}$. This corresponds to a breakthrough time of about $6 \mathrm{~min}$ for the current sample thickness of $\sim 2 \mathrm{~mm}$, which is the time needed for the first signal of hydrogen to be detected by the KP at the exit side (front/top surface), after hydrogen starts being loaded at the entry side (back/ bottom surface). 

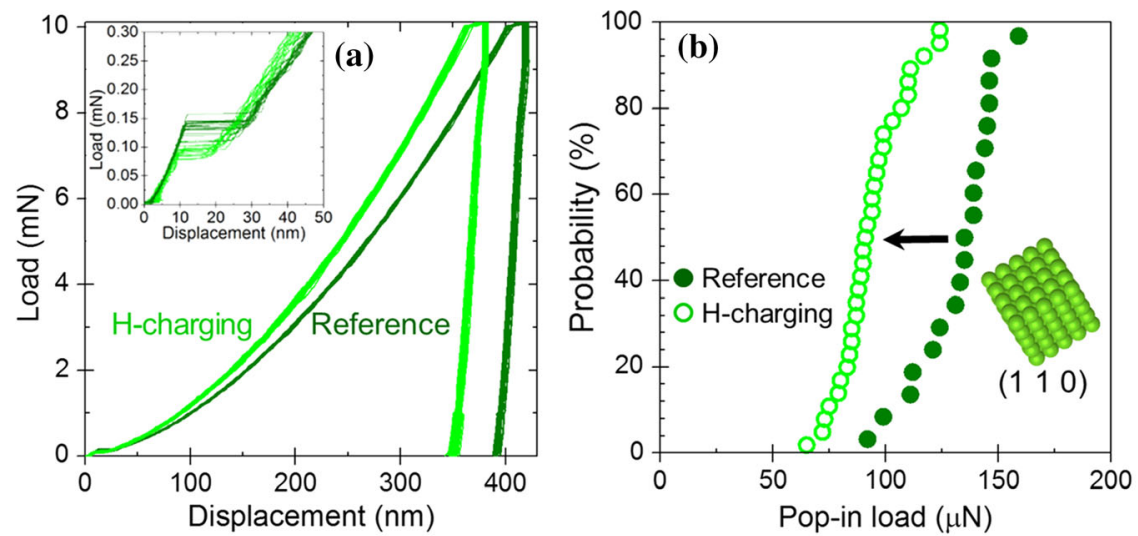

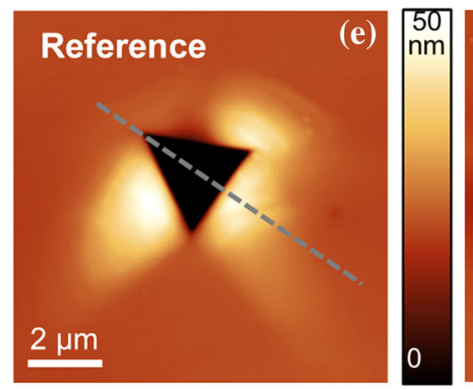
Figure 7 Back-side charging. (a) Nanoindentation load-
displacement curves and (b) probability distribution of the popin load for the $\left(\begin{array}{lll}1 & 1 & 0\end{array}\right)$ grain orientation $(\mathrm{Fe}-20 \mathrm{Cr})$, prior (dark green) and under hydrogen charging at $2 \mathrm{~mA} / \mathrm{cm}^{2}$ (light green). (c) Hardness and reduced modulus before and during hydrogen charging. (d) Changes in surface potential measured by KP at a

A signature of the stability of our approach is the continuous measurement of the elastic modulus. Although hardness tests usually show a similar hardening effect upon hydrogen charging, contradictory data have been reported regarding the influence of hydrogen in the elastic modulus, even using similar approaches as the one proposed here [44, 45]. The advantage of our concept is that the thickness of the sample $(2 \mathrm{~mm})$ and the characteristics of the charging cell (high compressive strength and no water absorption) make our measurements highly reproducible and stable. A negligible change of the reduced modulus and shear modulus was observed in simulations and experiments for bcc Fe at room temperature [46-48] and W [41]. In bcc Fe, this can be associated with the very limited solubility of hydrogen in the lattice. In addition, AFM maps of the indented surface, prior and during hydrogen charging, evidence that the surface remains pristine and, in this case, allow to monitor the increase in hardness
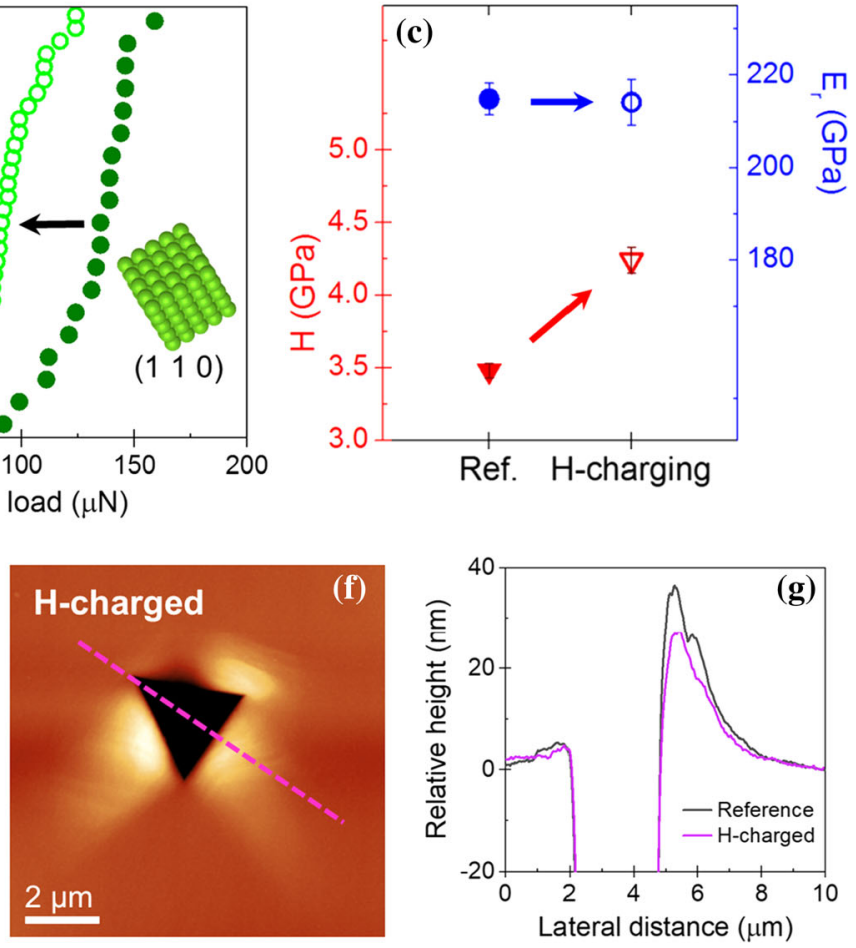

constant current of $2 \mathrm{~mA} / \mathrm{cm}^{2}$. (e) AFM maps of indents performed in air as reference and (f) after polarization at -1.45 $\mathrm{V}_{\text {ref }}$ for $8 \mathrm{~h}$ to promote hydrogen charging, followed by 13 days in a desiccator for hydrogen release. (g) Height profiles along the dashed lines in $\mathbf{d}$ ) and $\mathbf{e}$ ).

due to hydrogen by the reduced pile-ups and residual imprints (Fig. 7e-g).

According to our experience developing and using this new concept, we provide here some guidance for the use of the back-side charging approach and reliable data acquisition:

- As for the front-side charging, perform the frame stiffness calibration by directly mounting the reference material in the cell for hydrogen charging.

- The material used in the cell body is essential. For both, front-side and back-side charging, the cell body has to be built using a chemical-resistant material, with high compressive strength and no water absorption. The best compromise of these characteristics is in our opinion PCTFE, as explained before.

- The thickness of the sample has to ensure mechanical stability prior and during hydrogen charging. A thin sample introduces errors due to bending and stress generation, which are difficult 
to estimate once hydrogen is charged as it modifies the mechanical behavior. Find a compromise between the sample thickness and the charging time depending on the particular hydrogen diffusivity. An advantage of our back-side charging approach is that the use of a hydrogel allows stable charging conditions for at least 2 days. During this time, the cell can be mounted and unmounted from the nanoindenter stage without stopping the charging process and charging can be also restarted at any time by exchanging the electrolyte when needed.

- Use the electrolyte in a hydrogel form to promote a stable HER and avoid contact loss between the sample and the electrolyte due to $\mathrm{H}_{2(\mathrm{~g})}$ bubbles.

- Introduce an inert atmosphere around the sample to enable a faster equilibrium between the hydrogen absorption at the charging side and the desorption rates through the testing surface. This limits the formation of strong concentration gradients and ensures the presence of hydrogen in the testing region.

- Mirror polish also the charging side of the sample (back/bottom surface). A smooth surface provides stability and homogeneity during the charging process.

\section{Conclusions}

We presented a new approach to perform nanoindentation tests during hydrogen charging, referred here as back-side charging, where hydrogen is provided at the sample "back" surface and reaches the testing ("front") surface through bulk diffusion. A comparison is provided, for bcc FeCr alloys, to the established "electrochemical nanoindentation" approach, referred here as front-side charging, where the testing/front surface is directly charged through electrochemical processes. For this, two custom setups were designed and built in-house. A similar trend using both setups was found in the pop-in analysis, indicating a reduction of the shear stress for dislocation nucleation. However, an increase in hardness and negligible change in the elastic modulus was quantified using the back-side charging approach (in agreement with simulations and ex-situ experiments in bcc materials), while a decrease in hardness and elastic modulus was suggested by the front-side charging (attributed here to surface evolution during hydrogen charging). AFM revealed that the surface topography might change substantially during front-side charging, explaining the main differences observed in the nanoindentation results with respect to back-side charging. In accordance, some guidelines were presented for a more reliable use of either approach, and their advantages and disadvantages are summarized here below.

\section{Front-side charging}

- Advantages: fast charging of the testing surface, reducing concentration gradients in the tested volume.

- Disadvantages: the main disadvantage is related to surface evolution/damage during charging. This limits the charging electrolytes that can be used and the testing time to short periods. The sample has to be re-polish between tests and often post analyses of slip traces or dislocation structures at the surface are not possible in sensitive samples such as our FeCr alloys.

\section{Back-side charging}

- Advantages: as the testing surface is not affected/damaged by the charging process, the measurements present very high reproducibility and repeatability, while the observed phenomena can be attributed solely to hydrogen. Post analyses are also reliable. The method can be exploited to perform time-dependent measurements considering hydrogen absorption and diffusion through the material, which will be shown in a further publication, and used for different types of micromechanical tests.

- Disadvantages: the main disadvantages are related to a long time necessary for hydrogen to reach the testing side in alloys with low hydrogen diffusivity, such as fcc metals, and hydrogen desorption if an oxygen-free environment is not provided.

\section{Acknowledgements}

This work was supported by the Deutsche For schungsgemeinschaft (DFG) under the project DU 1628/1-1 and the European Research Council (ERC), 
grant no. 787446-GB-CORRELATE. We are grateful for the support of A. Kostka in performing EBSD analyses and helpful discussion, as well as $\mathrm{M}$. Rohwerder for his advice in KP.

\section{Funding}

Open Access funding enabled and organized by Projekt DEAL.

\section{Data availability}

The data that support the findings of this study are available from the corresponding authors upon reasonable request.

\section{Compliance with ethical standards}

Conflict of interest No potential conflict of interest was reported by the author(s).

Open Access This article is licensed under a Creative Commons Attribution 4.0 International License, which permits use, sharing, adaptation, distribution and reproduction in any medium or format, as long as you give appropriate credit to the original author(s) and the source, provide a link to the Creative Commons licence, and indicate if changes were made. The images or other third party material in this article are included in the article's Creative Commons licence, unless indicated otherwise in a credit line to the material. If material is not included in the article's Creative Commons licence and your intended use is not permitted by statutory regulation or exceeds the permitted use, you will need to obtain permission directly from the copyright holder. To view a copy of this licence, visit http://creativecommons.org/licen ses/by/4.0/.

\section{References}

[1] Wang YJ, Fang B, Wang X et al (2018) Recent advancements in the development of bifunctional electrocatalysts for oxygen electrodes in unitized regenerative fuel cells (URFCs). Prog Mater Sci 98:108-167. https://doi.org/10.10 16/j.pmatsci.2018.06.001
[2] Sorensen B (2012) Fuel Cells.3.1 Basic concepts. Hydrogen Fuel Cells. https://doi.org/10.1016/B978-0-12-387709-3.50 003-6

[3] Troiano AR (2016) The Role of Hydrogen and Other Interstitials in the Mechanical Behavior of Metals: (1959 Edward De Mille Campbell Memorial Lecture). Metallogr Microstruct Anal 5:557-569. https://doi.org/10.1007/s13632-0160319-4

[4] Tiwari GP, Bose A, Chakravartty JK et al (2000) A study of internal hydrogen embrittlement of steels. Mater Sci Eng A 286:269-281. https://doi.org/10.1016/S0921-5093(00)0079 3-0

[5] Lynch S (2012) Hydrogen embrittlement phenomena and mechanisms. Corros Rev 30:105-123. https://doi.org/10.15 15/corrrev-2012-0502

[6] Eliaz N, Shachar A, Tal B, Eliezer D (2002) Characteristics of hydrogen embrittlement, stress corrosion cracking and tempered martensite embrittlement in high-strength steels. Eng Fail Anal 9:167-184. https://doi.org/10.1016/S1350-63 07(01)00009-7

[7] Song J, Curtin WA (2013) Atomic mechanism and prediction of hydrogen embrittlement in iron. Nat Mater 12:145-151. h ttps://doi.org/10.1038/nmat3479

[8] Shih DS, Robertson IM, Birnbaum HK (1988) Hydrogen embrittlement of $\alpha$ titanium: in situ tem studies. Acta Metall 36:111-124. https://doi.org/10.1016/0001-6160(88)90032-6

[9] Oriani RA, Josephic PH (1974) Equilibrium aspects of hydrogen-induced cracking of steels. Acta Metall 22:1065-1074. https://doi.org/10.1016/0001-6160(74)90061 $-3$

[10] Birnbaum HK, Sofronis P (1994) Hydrogen-enhanced localized plasticity-a mechanism for hydrogen-related fracture. Mater Sci Eng A 176:191-202. https://doi.org/10.1016/ 0921-5093(94)90975-X

[11] Robertson IM (1999) The effect of hydrogen on dislocation dynamics. Eng Fract Mech 64:649-673. https://doi.org/10. 1016/S0013-7944(99)00094-6

[12] Sofronis P, Robertson IM (2002) Transmission electron microscopy observations and micromechanical/continuum models for the effect of hydrogen on the mechanical behaviour of metals. Philos Mag A Phys Condens Matter, Struct Defects Mech Prop 82:3405-3413. https://doi.org/10.1080/ 01418610208240451

[13] Tal-Gutelmacher E, Gemma R, Volkert CA, Kirchheim R (2010) Hydrogen effect on dislocation nucleation in a vanadium (lll 100$)$ single crystal as observed during nanoindentation. Scr Mater 63:1032-1035. https://doi.org/10.1016/ j.scriptamat.2010.07.039

[14] Katz Y, Tymiak N, Gerberich WW (2001) Nanomechanical probes as new approaches to hydrogen/deformation 
interaction studies. Eng Fract Mech 68:619-646. https://doi. org/10.1016/S0013-7944(00)00119-3

[15] Barnoush A, Vehoff H (2006) Electrochemical nanoindentation: A new approach to probe hydrogen/deformation interaction. Scr Mater 55:195-198. https://doi.org/10.1016/j. scriptamat.2006.03.041

[16] Fang X, Kreter A, Rasinski M et al (2018) Hydrogen embrittlement of tungsten induced by deuterium plasma: insights from nanoindentation tests. $\mathrm{J}$ Mater Res 33:3530-3536. https://doi.org/10.1557/jmr.2018.305

[17] Barnoush A, Kheradmand N, Hajilou T (2015) Correlation between the hydrogen chemical potential and pop-in load during in situ electrochemical nanoindentation. Scr Mater 108:76-79. https://doi.org/10.1016/j.scriptamat.2015.06.021

[18] Barnoush A, Dake J, Kheradmand N, Vehoff H (2010) Examination of hydrogen embrittlement in FeAl by means of in situelectrochemical micropillar compression and nanoindentation techniques. Intermetallics 18:1385-1389. https://d oi.org/10.1016/j.intermet.2010.01.001

[19] Gaspard V, Kermouche G, Delafosse D, Barnoush A (2014) Hydrogen effect on dislocation nucleation in a ferritic alloy $\mathrm{Fe}-15 \mathrm{Cr}$ as observed per nanoindentation. Mater Sci Eng A 604:86-91. https://doi.org/10.1016/j.msea.2014.02.041

[20] Kirchheim R (2010) Revisiting hydrogen embrittlement models and hydrogen-induced homogeneous nucleation of dislocations. Scr Mater 62:67-70. https://doi.org/10.1016/j. scriptamat.2009.09.037

[21] Duarte MJ, Fang X, Brinckmann S, Dehm G (2018) New approaches for in-situ nanoindentation of hydrogen charged alloys: insights on bec FeCr alloys. In: Verh DPG, 2018. h ttps://www.dpg-verhandlungen.de/year/2018/conference/berl in/part $/ \mathrm{mm} / \mathrm{session} / 3 /$ contribution $/ 8$.

[22] Grabke HJ, Riecke E (2000) Absorption and diffusion of hydrogen in steels. Mater Tehnol 34:331-342

[23] Lucas BN, Oliver WC (1999) Indentation power-law creep of high-purity indium. Metall Mater Trans A 30:601-610. h ttps://doi.org/10.1007/s11661-999-0051-7

[24] Epler E (2015) Mechanische Eigenschaften von Lithiumionen Batterieelektrodenmaterialien bei verschiedenen Ladezuständen. $\mathrm{PhD}$ Dissertation, Georg-August-Universität Göttingen

[25] Evers S, Senöz C, Rohwerder M (2013) Hydrogen detection in metals: A review and introduction of a Kelvin probe approach. Sci Technol Adv Mater 14(1):014201. https://doi. org/10.1088/1468-6996/14/1/014201

[26] Wu CH, Krieger W, Rohwerder M (2019) On the robustness of the Kelvin probe based potentiometric hydrogen electrode method and its application in characterizing effective hydrogen activity in metal: 5 wt. \% Ni cold-rolled ferritic steel as an example. Sci Technol Adv Mater 20:1073-1089. https://doi.org/10.1080/14686996.2019.1687255

[27] Hamann CH, Hamnett A, Vielstich W (2007) Electrochemistry. Wiley, Hoboken

[28] Gerberich WW, Nelson JC, Lilleodden ET et al (1996) Indentation induced dislocation nucleation: the initial yield point. Acta Mater 44:3585-3598. https://doi.org/10.1016/1 359-6454(96)00010-9

[29] Bahr DF, Kramer DE, Gerberich WW (1998) Non-linear deformation mechanisms during nanoindentation. Acta Mater 46:3605-3617. https://doi.org/10.1016/S1359-6454(9 8)00024-X

[30] Schuh CA, Lund AC (2004) Application of nucleation theory to the rate dependence of incipient plasticity during nanoindentation. J Mater Res 19:2152-2158. https://doi.org/ 10.1557/JMR.2004.0276

[31] Narayanan S, McDowell DL, Zhu T (2014) Crystal plasticity model for BCC iron atomistically informed by kinetics of correlated kinkpair nucleation on screw dislocation. J Mech Phys Solids 65:54-68. https://doi.org/10.1016/j.jmps.2014. 01.004

[32] Johnson KL (2013) Normal contact of elastic solids - Hertz theory. In: Contact Mechanics. Cambridge University Press, pp 62-106

[33] Hertz H (1881) On the contact of elastic solids. J Reine Angew Math 92:156-171

[34] Valtiner M, Ankah GN, Bashir A, Renner FU (2011) Atomic force microscope imaging and force measurements at electrified and actively corroding interfaces: Challenges and novel cell design. Rev Sci Instrum 82:023703. https://doi. org/10.1063/1.3541650

[35] Deng H, Reynolds CT, Cabrera NO et al (2010) The water absorption behaviour of all-polypropylene composites and its effect on mechanical properties. Compos Part B Eng 41:268-275. https://doi.org/10.1016/j.compositesb.2010.02. 007

[36] Oliver WC, Pharr GM (1992) An improved technique for determining hardness and elastic modulus using load and displacement sensing indentation experiments. J Mater Res 7:1564-1583. https://doi.org/10.1557/JMR.1992.1564

[37] Kheradmand N, Johnsen R, Olsen JS, Barnoush A (2016) Effect of hydrogen on the hardness of different phases in super duplex stainless steel. Int $\mathrm{J}$ Hydrogen Energy 41:704-712. https://doi.org/10.1016/j.ijhydene.2015.10.106

[38] Wang D, Lu X, Deng Y et al (2019) Effect of hydrogen on nanomechanical properties in Fe-22Mn-0.6C TWIP steel revealed by in-situ electrochemical nanoindentation. Acta Mater 166:618-629. https://doi.org/10.1016/j.actamat.2018. 12.055 
[39] Mertens G, Duprez L, De Cooman BC, Verhaege M (2007) Hydrogen absorption and desorption in steel by electrolytic charging. Adv Mater Res 15-17:816-821. https://doi.org/10. 4028/www.scientific.net/amr.15-17.816

[40] Leyson GPM, Grabowski B, Neugebauer J (2016) Multiscale modeling of hydrogen enhanced homogeneous dislocation nucleation. Acta Mater 107:144-151. https://doi. org/10.1016/j.actamat.2016.01.036

[41] Fang X, Rasinski M, Kreter A et al (2019) Plastic deformation of tungsten due to deuterium plasma exposure: insights from micro-compression tests. Scr Mater 162:132-135. https://doi.org/10.1016/j.scriptamat.2018.10. 052

[42] Cottrell AH, Jaswon MA (1949) Distribution of solute atoms round a moving dislocation. Proc $\mathrm{R}$ Soc Lond A 199:104-114. https://doi.org/10.1016/j.msea.2005.03.046

[43] Delafosse D (2012) Gaseous Hydrogen Embrittlement on Materials in Energy Technologies: Mechanisms, Modelling and Future Developments. In: Hydrogen effects on the plasticity of face centred cubic (fcc) crystals. Woodhead Publishing Limited, pp 247-285. https://doi.org/10.1533/97 80857095374.2.247
[44] Müller C, Zamanzade M, Motz C (2019) The impact of hydrogen on mechanical properties; A new in situ nanoindentation testing method. Micromachines 10:114. https://doi. org/10.3390/mi10020114

[45] Kim J, Tasan CC (2019) Microstructural and micro-mechanical characterization during hydrogen charging: an in situ scanning electron microscopy study. Int J Hydrogen Energy 44:6333-6343. https://doi.org/10.1016/j.ijhydene.20 18.10 .128

[46] Zhang TY, Jiang FX, Chu WY, Hsiao CM (1985) Effect of hydrogen on the Young's modulus of iron. Metall Trans A 16:1655-1662. https://doi.org/10.1007/BF02663021

[47] Nagumo M (2016) Fundamentals of Hydrogen Embrittlement. Springer, New York. https://www.springer.com/de/bo ok/9789811001604. Accessed 9 Jun 2020

[48] Lunarska E, Zielinski A, Smialowski M (1977) Effect of hydrogen on shear modulus of polycrystalline $\alpha$ iron. Acta Metall 25:305-308. https://doi.org/10.1016/0001-6160(77)9 0149-3

Publisher's Note Springer Nature remains neutral with regard to jurisdictional claims in published maps and institutional affiliations. 